\title{
COMPOSIÇÃO FLORÍSTICA E ESTRUTURA DE UM FRAGMENTO FLORESTAL EM ÁREA ECOTONAL CERRADO-PANTANAL
}

\author{
Oacy Eurico de Oliveira ${ }^{1}$, Thelma Shirlen Soares ${ }^{2}$, Reginaldo Brito da Costa ${ }^{3}$ \\ ${ }^{1}$ Instituto Federal de Educação, Ciência e Tecnologia de Mato Grosso - Campus São Vicente, Santo Antônio do Leverger - MT, \\ E-mail: oacyeurico@gmail.com \\ ${ }^{2}$ Universidade Federal de Goiás - Regional Jataí, Jataí - GO, E-mail: thelmasoares@ufg.br \\ ${ }^{3}$ Universidade Católica Dom Bosco, Campo Grande - MS, E-mail: reg.brito.costa@gmail.com
}

\section{RESUMO}

Este estudo objetivou avaliar a florística e a fitossociologia dos indivíduos arbóreos e arbustivos de um fragmento florestal em Santo Antônio do Leverger-MT, em área ecotonal cerrado e pantanal. Em vinte e cinco parcelas de 20 x 20m, área amostral de 1,0 ha, foram inventariados todos os indivíduos com $C A P \geq 15 \mathrm{~cm}$. Registrou-se 37 famílias, 61 gêneros, 77 espécies, 1 família indeterminada e 1 espécie não identificada. Destacaram-se em número de espécies, as famílias Fabaceae, Annonaceae, Sapotaceae, Apocynaceae, Cecropiaceae, Myrtaceae, Burseraceae, Melastomataceae, Moraceae e Rubiaceae. A espécie de maior índice de valor de importância (IVI) foi Ocotea olivacea (Lauraceae). A área apresentou densidade de 695 indivíduos/ha e área basal de 20,3 m²/ha. O índice de Shannon-Weaver e equabilidade de Pielou encontrados foram 3,81 e 0,87, respectivamente, evidenciando uma comunidade com alta diversidade.

Palavras-chave: fitossociologia, transição, comunidade arbórea

\section{STRUCTURE AND FLORISTIC COMPOSITION OF A FOREST FRAGMENT IN AN ECOTONAL AREA SAVANNAH-PANTANAL}

\begin{abstract}
This study aimed to evaluate the floristic and the phytosociology of trees and shrubs, in an ecotonal area between cerrado and pantanal. In twenty-five plots of $20 \times 20 \mathrm{~m}$, sample area of 1.0 ha, all individuals with $\mathrm{CBH} \geq 15.00 \mathrm{~cm}$ were inventoried. We registered 37 families, 61 genera and 77 species, 1 indeterminated family and 1 unidentified species. The families Fabaceae, Annonaceae, Sapotaceae, Apocynaceae, Cecropiaceae, Myrtaceae, Burseraceae, Melastomataceae, Moraceae and Rubiaceae stood out in number of species. The species with the highest Importance Value Index (IVI) was Ocotea olivacea (Lauraceae). The area had a density of 695 individuals/ha and the basal area of $20.3 \mathrm{~m}^{2} / \mathrm{ha}$. The index of Shannon-weaver and Evenness values were 3.81 and 0.87 , respectively, indicating a community with high diversity.
\end{abstract}

Keywords: phytosociology, transition, community trees 


\section{COMPOSIÇÃO FLORÍSTICA E ESTRUTURA DE UM FRAGMENTO FLORESTAL EM ÁREA \\ ECOTONAL CERRADO-PANTANAL}

\section{INTRODUÇÃO}

O Cerrado é o segundo maior bioma brasileiro, localizado no Brasil central, com área $2.036 .448 \mathrm{~km}^{2}$ correspondendo à área total do Brasil em 23,92\% (IBGE, 2004) e o Pantanal é uma planície sedimentar com aproximadamente $140.000 \mathrm{~km}^{2}$ localizada na Bacia do Rio Paraguai (GUARIM et al., 2000).

De acordo com Neiff (2003), o ecótono é uma área de tensão instável sem padrões próprios de variabilidade e, portanto, de diversidade, e cuja estrutura depende inteiramente das tensões de duas comunidades adjacentes. Para Odum (2010), é uma zona de união ou um cinturão de tensão que pode conter organismos de cada uma das comunidades que se sobrepõem, além dos organismos característicos.

Embora os diferentes tipos de vegetação no Brasil estejam teoricamente bem definidos, com base em critérios florísticos, fisionômicos e ecológicos (VELOSO et al., 1991), mapear e classificar as vegetações localizadas nas áreas de transição entre os biomas brasileiros não é tarefa simples (HAIDAR et al., 2013).

Estudos sobre a estrutura, dinâmica e composição dos diferentes grupos biológicos nas regiões de transições que se instalam entre os biomas cerrado e pantanal ainda são pouco representativos.

O conhecimento da biodiversidade das formações vegetais é a condição primária e fundamental para $\mathrm{o}$ desenvolvimento não só de investigações botânicas e ecológicas, mas, sobretudo para o estabelecimento de modelos de preservação e conservação dos ecossistemas (MORELLATO \& LEITÃO FILHO, 1995; FERREIRA JÚNIOR et al., 2008).

Estudos florísticos e fitossociológicos têm fornecido informações importantes para a compreensão dos padrões biogeográficos, e subsidiado a delimitação de áreas prioritárias para a conservação de recursos fitogenéticos (FELFILI et al., 2002).

Portanto, o conhecimento das espécies, torna-se relevante na caracterização da vegetação que ocorrem em fragmentos de ecótonos, neste caso, cerradopantanal, subsidiando práticas de manejo florestal, manutenção da biodiversidade, educação ambiental e diretrizes para a preservação e conservação dessas áreas.

Este estudo objetivou avaliar a composição florística e a fitossociologia da comunidade arbórea e arbustiva que em fragmentos na área ecotonal dos biomas cerrado e pantanal, no município de Santo Antônio do Leverger - MT. 


\section{MATERIAL E MÉTODOS}

A área de estudo refere-se a um fragmento florestal de 74,00 ha que apresenta alguns aspectos peculiares e intrínsecos: situa-se na fase de transição cerrado sensu stricto e cerrado sensu lato; entre os biomas Cerrado e Pantanal; e divisa das bacias hidrográficas: Bacia do Prata e Bacia Araguaia. Essa área pertence ao Instituto Federal de Educação, Ciência e Tecnologia de Mato Grosso (IFMT), Campus São Vicente, município de Santo Antônio do Leverger - MT, localizado nas coordenadas geográficas: $15^{\circ} 49^{\prime} 21^{\prime \prime} \mathrm{S}$ e $55^{\circ} 25^{\prime} 06^{\prime}$ W. O clima é caracterizado como tropical (Aw), segundo a classificação de Köppen e apresenta duas estações bem definidas: uma quente e úmida e outra mais fria e seca com déficit hídrico. A temperatura anual média está em torno de 22 ${ }^{\circ} \mathrm{C}$ e precipitação média anual é de 1.860 $\mathrm{mm}$.

Os solos são classificados como Podzólico Vermelho Escuro Distrófico (Argissolo Vermelho Distrófico) e Latossolo Vermelho Escuro Distrófico (Latossolo Vermelho Distrófico) associados às Areias Quartzosas, que se relacionam especialmente às coberturas terciárias desenvolvidas sobre arenito de formação furnas, com relevo plano a suave ondulado (BRASIL, 1982).
O método de amostragem utilizado foi o de parcelas (MUELLER-DOMBOIS \& ELLENBERG, 1974). Foram alocadas 25 parcelas de $20 \times 20 \mathrm{~m}\left(400 \mathrm{~m}^{2}\right)$, perfazendo um total de $10.000 \mathrm{~m}^{2} \quad(1 \mathrm{ha})$. Na amostragem, foram incluídos todos os indivíduos arbóreos-arbustivos com circunferência a $1,3 \mathrm{~m}$ do solo $(C A P) \geq 15$ $\mathrm{cm}$.

A identificação taxonômica das espécies foi efetuada mediante consultas a herbário, consultas a especialistas e por meio de literatura especializada. A sinonímia e a grafia dos taxa foram confirmadas e atualizadas mediante consulta ao banco de dados da Lista de Espécies da Flora do Brasil (BRASIL, 2016). O sistema de classificação taxonômica adotado foi APG IV (APG, 2016). O material botânico coletado em estado reprodutivo está depositado no Herbário Central da Universidade Federal de Mato Grosso (HCUFMT).

Para a caracterização florística da área, foram utilizados os índices de diversidade: Shannon-Weaver (H') e equabilidade de Pielou (J'). A listagem florística foi elaborada a partir da amostragem fitossociológica. A análise fitossociológica envolveu as estimativas dos parâmetros estruturas horizontal e vertical de 


\section{COMPOSIÇÃO FLORÍSTICA E ESTRUTURA DE UM FRAGMENTO FLORESTAL EM ÁREA \\ ECOTONAL CERRADO-PANTANAL}

modo a conhecer a importância de cada espécie na comunidade. Os parâmetros da estrutura horizontal calculados foram: densidade relativa, dominância relativa, frequência relativa e índices de valor de importância e de cobertura (MUELLERDOMBOIS \& ELLENBERG, 1974). O parâmetro da estrutura vertical calculado foi a posição sociológica relativa conforme Scolforo \& Mello (1997).

\section{RESULTADOS E DISCUSSÃO}

O levantamento florístico registrou 695 indivíduos, distribuídos em 37 famílias, 61 gêneros e 76 espécies, sendo que 4 espécies foram identificadas apenas em nível de família (Tabela 1). As famílias que apresentaram maior riqueza florística foram: Fabaceae com 12 espécies, Annonaceae e Sapotaceae (5 espécies cada), Apocynaceae, Myrtaceae e Urticaceae (4 espécies cada), Burseraceae, Melastomataceae, Moraceae e Rubiaceae (3 espécies cada). Essas famílias contribuíram com $60,5 \%$ do total das espécies amostradas. As famílias Araliaceae, Chrysobalanaceae, Lauraceae, Malvaceae e Primulaceae apresentaram duas espécies cada. Vinte famílias $(26,3 \%)$ foram representadas por uma única espécie.

Os resultados confirmam informações de Silveira et al. (2009) e Silva et al. (2016) que também encontraram as mesmas famílias, sendo as mais bem representadas em estudos no cerrado matogrossense e de Morais et al. (2013) e Rodrigues et al. (2015) estudando a composição florística no pantanal matogrossense. $\mathrm{O}$ índice de diversidade de Shannon (H') encontrado de 3,81 nats.indivíduos ${ }^{-1}$, pode ser considerado alto, quando comparando à média para os biomas Cerrado e Pantanal. Isto pode estar relacionado ao fato de se encontrar em uma área de transição. Foi observado o valor de 0,87 para equabilidade de Pielou. Resultados similares foram encontrados na região leste de Mato Grosso, de 0,87 e 0,84 (MARIMON JÚNIOR \& HARIDASAN, 2005), indicando alta uniformidade nas proporções do número de indivíduos/número de espécies dentro da comunidade florestal.

A área apresentou densidade absoluta de 694 indivíduos.ha ${ }^{-1}$ e área basal de 20 $\mathrm{m}^{2} \cdot \mathrm{ha}^{-1}$ (Tabela 2). 
Tabela 1 Relação das famílias e espécies, com seus respectivos nomes populares, amostradas na área do córrego da cabeceira do Rio das Mortes em área ecotonal Cerrado-Pantanal, município de Santo Antônio do Leverger- MT, 2011.

\begin{tabular}{|c|c|c|}
\hline Família & Espécie & Nome Popular \\
\hline Anacardiaceae & Tapirira guianensis Aubl. & Pombeiro \\
\hline Annonaceae & $\begin{array}{l}\text { Unonopsis } \mathrm{sp} . \\
\text { Unonopsis guatterioides (A.DC.) R.E.Fr. } \\
\text { Xylopia aromatica (Lam.) Mart. } \\
\text { Xylopia benthamii } \text { R.E.Fr. } \\
\text { Xylopia emarginata } \text { Mart. }\end{array}$ & $\begin{array}{l}\text { Calonga } \\
\text { Carvoeiro-emborrachado } \\
\text { Pindaíba } \\
\text { Pindaíba-folha-larga } \\
\text { Pimenta-de-macaco }\end{array}$ \\
\hline Apocynaceae & $\begin{array}{l}\text { Himatanthus lancifolius (Müll. Arg.) Woodson } \\
\text { Himatanthus sucuubus (Spruce ex Müll.Arg.) Woodson } \\
\text { Apocynaceae } 1 \\
\text { Apocynaceae } 2\end{array}$ & $\begin{array}{l}\text { Angélica } \\
\text { Angélica } \\
\text { Peroba } \\
\text { Peroba-do-brejo }\end{array}$ \\
\hline Araliaceae & $\begin{array}{l}\text { Schefflera morototoni (Aubl.) Maguire et al. } \\
\text { Schefflera vinosa (Cham. \& Schltdl.) Frodin \& Fiaschi. }\end{array}$ & $\begin{array}{l}\text { Mandiocão } \\
\text { Mandiocão }\end{array}$ \\
\hline Bignoniaceae & Jacaranda copaia (Aubl.) D. Don & Pinho \\
\hline Boraginaceae & Cordia alliodora (Ruiz \& Pav.) Cham. & Louro-branco \\
\hline Burseraceae & $\begin{array}{l}\text { Protium heptaphyllum (Aubl.) Marchand } \\
\text { Protium pilosissimum Engl. } \\
\text { Protium pilosum (Cuatrec.) Daly }\end{array}$ & $\begin{array}{l}\text { Amescla } \\
\text { Laranjinha } \\
\text { Amesclinha }\end{array}$ \\
\hline Calophyllaceae & Calophyllum brasiliense Cambess. & Guanandi \\
\hline Chrysobalanaceae & $\begin{array}{l}\text { Licania humilis Cham. \& Schltdl. } \\
\text { Licania micrantha Miq. }\end{array}$ & $\begin{array}{l}\text { Sassafrás } \\
\text { Pau-terra }\end{array}$ \\
\hline Combretaceae & Buchenavia tomentosa Eichler & Mirindiba \\
\hline Ebenaceae & Diospyros guianensis (Aubl.) Gürke & Olho-de-boi \\
\hline Elaeocarpaceae & Sloanea guianensis (Aubl.) Benth. & Pateiro \\
\hline Erythroxylaceae & Erythroxylum sp. & Carvoeiro-do-brejo \\
\hline Euphorbiaceae & Mabea fistulifera Mart. & Fedegoso \\
\hline Fabaceae & $\begin{array}{l}\text { Abarema jupunba (Willd.)Britton \& Killp. } \\
\text { Apuleia leiocarpa (Vogel) J.F.Macbr. } \\
\text { Bauhinia sp. } \\
\text { Copaifera langsdorffii Desf. } \\
\text { Enterolobium schomburgkii (Benth.) Benth. } \\
\text { Enterolobium timbouva Mart. } \\
\text { Inga sp. } \\
\text { Inga vera Willd. } \\
\text { Ormosia arborea (Vell.) Harms } \\
\text { Tachigali aurea Tul. } \\
\text { Tachigali rubiginosa (Mart. ex Tul.) Oliveira-Filho } \\
\text { Fabaceae } 1\end{array}$ & $\begin{array}{l}\text { Pindaíba-folha-miúda } \\
\text { Garapeira } \\
\text { Pata-de-vaca } \\
\text { Pau-de-óleo } \\
\text { Farinha-seca } \\
\text { Orelha-de-macaco } \\
\text { Ingá } \\
\text { Ingá } \\
\text { Folha-parda } \\
\text { Carvoeiro } \\
\text { Jacarezinho } \\
\text { Pau-ferro }\end{array}$ \\
\hline Humiriaceae & Sacoglottis matogrossensis Malme & Pau-serra \\
\hline Lacistemataceae & Lacistema polystachium Schnizl. & Calonga \\
\hline Lauraceae & $\begin{array}{l}\text { Lauraceae } 1 \\
\text { Ocotea olivacea A.C.Sm. }\end{array}$ & $\begin{array}{l}\text { Canela-do-brejo } \\
\text { Caneleiro }\end{array}$ \\
\hline Lythraceae & Physocalymma scaberrimum Pohl & Aricá \\
\hline Malpighiaceae & Byrsonima spicata (Cav.) DC. & Murici \\
\hline
\end{tabular}




\section{COMPOSIÇÃO FLORÍSTICA E ESTRUTURA DE UM FRAGMENTO FLORESTAL EM ÁREA ECOTONAL CERRADO-PANTANAL}

Tabela 1. Relação das famílias e espécies, com seus respectivos nomes populares, amostradas na área do córrego da cabeceira do Rio das Mortes em área ecotonal Cerrado-Pantanal, município de Santo Antônio do Leverger- MT, 2011.

\begin{tabular}{|c|c|c|}
\hline Família & Espécie & Nome Popular \\
\hline Malvaceae & $\begin{array}{l}\text { Eriotheca gracilipes (K.Schum.) A.Robyns } \\
\text { Luehea divaricata Mart. \& Zucc. }\end{array}$ & $\begin{array}{l}\text { Paineira } \\
\text { Açoita-cavalo }\end{array}$ \\
\hline \multirow{3}{*}{ Melastomataceae } & Miconia cuspidata Naudin & $\begin{array}{l}\text { Fedegoso-pintado-casca- } \\
\text { lisa }\end{array}$ \\
\hline & Miconia ferruginata DC. & $\begin{array}{l}\text { Folha-parda-folha- } \\
\text { ferrugínea }\end{array}$ \\
\hline & Miconia sp. & Folha-parda-folha-larga \\
\hline \multirow{3}{*}{ Moraceae } & Brosimum rubescens Taub. & Alecrim \\
\hline & Ficus sp. & Figueira \\
\hline & Pseudolmedia laevignata Trécul. & Leiteiro \\
\hline Myristicaceae & Virola sebifera Aubl. & Pimenta-de-macaco \\
\hline \multirow{4}{*}{ Myrtaceae } & $\begin{array}{l}\text { Campomanesia eugenioides (Cambess.) D.Legrand ex } \\
\text { Landrum }\end{array}$ & Orvalheira \\
\hline & Eugenia sp. & Goiabinha \\
\hline & Myrcia fallax (Rich.) DC. & Cascudinho \\
\hline & Myrcia guianensis (Aubl.) DC. & Cascudinho \\
\hline Phyllanthaceae & Hyeronima alchorneoides Allemão & Araputanga \\
\hline Piperaceae & Piper sp. & Jaborandi \\
\hline Primulaceae & $\begin{array}{l}\text { Myrsine coriacea (Sw.) R. Br. ex Roem. \& Schult. } \\
\text { Myrsine umbellata Mart. }\end{array}$ & $\begin{array}{l}\text { Pururuca } \\
\text { Pororoca }\end{array}$ \\
\hline Proteaceae & Roupala brasiliensis Klotzsch & Coração-de-negro \\
\hline Rubiaceae & $\begin{array}{l}\text { Cordiera edulis (Rich.) Kuntze } \\
\text { Cordiera macrophylla (K. Schum.) Kuntze } \\
\text { Duroia duckei Huber. }\end{array}$ & $\begin{array}{l}\text { Marmelada } \\
\text { Marmelada } \\
\text { Marmelada }\end{array}$ \\
\hline Sapindaceae & Matayba guianensis Aubl. & Canjarana \\
\hline \multirow{5}{*}{ Sapotaceae } & $\begin{array}{l}\text { Chrysophyllum gonocarpum (Mart. \& Eichler ex Miq.) } \\
\text { Engl. }\end{array}$ & Rapadura \\
\hline & Ecclinusa ramiflora Mart. & Leiteiro-folha-larga \\
\hline & Manilkara sp. & Pintado-casca-grossa \\
\hline & Micropholis sp. & Chumbinho \\
\hline & Pouteria sp. & Ovinha \\
\hline Siparunaceae & Siparuna guianensis Aubl. & Negramina \\
\hline \multirow{4}{*}{ Urticaceae } & Cecropia hololeuca Miq. & Embaúba \\
\hline & Cecropia pachystachya Trécul & Embaúba \\
\hline & Pourouma cecropiifolia Mart. & Paineira \\
\hline & Pourouma sp. & Embaúba \\
\hline Vochysiaceae & Vochysia divergens Pohl & Cambará \\
\hline
\end{tabular}

Relativo à abundância o presente estudo foi inferior aos registrados em outros trabalhos na região, Felfili et al. (2002) e Silveira et al. (2009) cujos valores foram:
995; 1.282 e 848 indivíduos.ha ${ }^{-1}$. Todavia foi superior aos valores de dominância observados por esses autores, de 7,5; 18,06 e $6,3 \mathrm{~m}^{2} / \mathrm{ha}$, respectivamente. 
Tabela 2. Lista das espécies arbóreas ocorrentes na área do córrego da cabeceira Rio das Mortes (RM), em área Cerrado-Pantanal em ordem decrescente de índice de valor de importância (IVI\%), município de Santo Antônio do Leverger - MT, 2011.

\begin{tabular}{|c|c|c|c|c|c|c|c|c|}
\hline Espécie & $\mathbf{D A}$ & DR & DoA & DoR & FA & FR & IVI (\%) & IVC (\%) \\
\hline Ocotea olivacea A.C.Sm. & 67 & 9,65 & 1,13 & 5,64 & 68 & 4,51 & 6,60 & 7,65 \\
\hline Tapirira guianensis Aubl. & 23 & 3,31 & 1,66 & 8,31 & 56 & 3,71 & 5,11 & 5,81 \\
\hline Protium heptaphyllum (Aubl.) Marchand & 33 & 4,76 & 0,59 & 2,98 & 72 & 4,77 & 4,17 & 3,87 \\
\hline Physocalymma scaberrimum Pohl & 31 & 4,47 & 0,66 & 3,31 & 52 & 3,45 & 3,74 & 3,89 \\
\hline Sloanea guianensis (Aubl.) Benth. & 23 & 3,31 & 0,90 & 4,50 & 44 & 2,92 & 3,58 & 3,90 \\
\hline Buchenavia tomentosa Eichler & 4 & 0,58 & 1,74 & 8,74 & 16 & 1,06 & 3,46 & 4,66 \\
\hline Protium pilosissimum Engl. & 33 & 4,76 & 0,27 & 1,35 & 64 & 4,24 & 3,45 & 3,05 \\
\hline Cordia alliodora (Ruiz \& Pav.) Cham. & 19 & 2,74 & 0,81 & 4,06 & 48 & 3,18 & 3,33 & 3,40 \\
\hline Inga sp. & 25 & 3,60 & 0,66 & 3,31 & 36 & 2,39 & 3,10 & 3,46 \\
\hline Licania humilis Cham. \& Schltdl. & 24 & 3,46 & 0,64 & 3,21 & 32 & 2,12 & 2,93 & 3,33 \\
\hline Ormosia arborea (Vell.) Harms & 27 & 3,89 & 0,32 & 1,60 & 48 & 3,18 & 2,89 & 2,74 \\
\hline Inga vera Willd. & 17 & 2,45 & 0,54 & 2,72 & 40 & 2,65 & 2,61 & 2,58 \\
\hline Siparuna guianensis Aubl. & 27 & 3,89 & 0,09 & 0,45 & 44 & 2,92 & 2,42 & 2,17 \\
\hline Chrysophyllum gonocarpum (Mart. \& Eichler ex Miq.) Engl. & 15 & 2,16 & 0,43 & 2,18 & 40 & 2,65 & 2,33 & 2,17 \\
\hline Miconia sp. & 21 & 3,03 & 0,28 & 1,39 & 36 & 2,39 & 2,27 & 2,21 \\
\hline Jacaranda copaia (Aubl.) D. Don & 6 & 0,86 & 0,75 & 3,75 & 20 & 1,33 & 1,98 & 2,31 \\
\hline Roupala brasiliensis Klotzsch & 11 & 1,59 & 0,53 & 2,67 & 24 & 1,59 & 1,95 & 2,13 \\
\hline Cordiera edulis (Rich.) Kuntze & 20 & 2,88 & 0,19 & 0,95 & 28 & 1,86 & 1,90 & 1,92 \\
\hline Manilkara sp. & 5 & 0,72 & 0,74 & 3,70 & 16 & 1,06 & 1,83 & 2,21 \\
\hline Licania micrantha Miq. & 8 & 1,15 & 0,40 & 2,03 & 32 & 2,12 & 1,77 & 1,59 \\
\hline Miconia cuspidata Naudin & 11 & 1,59 & 0,36 & 1,78 & 28 & 1,86 & 1,74 & 1,68 \\
\hline Xylopia aromatica (Lam.) Mart. & 12 & 1,73 & 0,13 & 0,64 & 40 & 2,65 & 1,68 & 1,19 \\
\hline Myrsine coriacea (Sw.) R. Br. ex Roem. \& Schult. & 11 & 1,59 & 0,35 & 1,77 & 24 & 1,59 & 1,65 & 1,68 \\
\hline Himatanthus sucuubus (Spruce ex Müll.Arg.) Woodson & 8 & 1,15 & 0,31 & 1,53 & 32 & 2,12 & 1,60 & 1,34 \\
\hline Pouteria sp. & 12 & 1,73 & 0,28 & 1,38 & 24 & 1,59 & 1,57 & 1,56 \\
\hline Myrsine umbellata Mart. & 10 & 1,44 & 0,27 & 1,34 & 28 & 1,86 & 1,55 & 1,39 \\
\hline Copaifera langsdorffii Desf. & 1 & 0,14 & 0,71 & 3,56 & 4 & 0,27 & 1,32 & 1,85 \\
\hline Duroia duckei Huber. & 15 & 2,16 & 0,14 & 0,69 & 16 & 1,06 & 1,30 & 1,43 \\
\hline Pseudolmedia laevignata Trécul. & 9 & 1,30 & 0,13 & 0,65 & 28 & 1,86 & 1,27 & 0,97 \\
\hline Myrcia guianensis (Aubl.) DC. & 5 & 0,72 & 0,38 & 1,92 & 16 & 1,06 & 1,24 & 1,32 \\
\hline Tachigali aurea Tul. & 7 & 1,01 & 0,23 & 1,14 & 20 & 1,33 & 1,16 & 1,07 \\
\hline Myrcia fallax (Rich.) DC. & 10 & 1,44 & 0,13 & 0,65 & 20 & 1,33 & 1,14 & 1,04 \\
\hline Cordiera macrophylla (K. Schum.) Kuntze & 11 & 1,59 & 0,09 & 0,45 & 20 & 1,33 & 1,12 & 1,02 \\
\hline Miconia ferruginata DC. & 9 & 1,30 & 0,11 & 0,55 & 20 & 1,33 & 1,06 & 0,92 \\
\hline Abarema jupunba (Willd.)Britton \& Killp. & 7 & 1,01 & 0,10 & 0,49 & 24 & 1,59 & 1,03 & 0,75 \\
\hline Schefflera morototoni (Aubl.) Maguire et al. & 4 & 0,58 & 0,31 & 1,54 & 12 & 0,80 & 0,97 & 1,06 \\
\hline Eugenia sp. & 8 & 1,15 & 0,07 & 0,35 & 20 & 1,33 & 0,94 & 0,75 \\
\hline Tachigali rubiginosa (Mart. ex Tul.) Oliveira-Filho & 10 & 1,44 & 0,07 & 0,35 & 12 & 0,80 & 0,86 & 0,89 \\
\hline Lauraceae 1 & 1 & 0,14 & 0,42 & 2,08 & 4 & 0,27 & 0,83 & 1,11 \\
\hline Byrsonima spicata (Cav.) DC. & 4 & 0,58 & 0,22 & 1,09 & 12 & 0,80 & 0,82 & 0,83 \\
\hline Ficus sp. & 3 & 0,43 & 0,24 & 1,19 & 12 & 0,80 & 0,81 & 0,81 \\
\hline Sacoglottis matogrossensis Malme & 8 & 1,15 & 0,07 & 0,35 & 12 & 0,80 & 0,76 & 0,75 \\
\hline Mabea fistulifera Mart. & 3 & 0,43 & 0,21 & 1,04 & 12 & 0,80 & 0,76 & 0,73 \\
\hline Xylopia benthamii R.E.Fr. & 6 & 0,86 & 0,08 & 0,40 & 12 & 0,80 & 0,69 & 0,63 \\
\hline Fabaceae 1 & 5 & 0,72 & 0,05 & 0,25 & 16 & 1,06 & 0,68 & 0,49 \\
\hline Schefflera vinosa (Cham. \& Schltdl.) Frodin \& Fiaschi. & 2 & 0,29 & 0,22 & 1,08 & 8 & 0,53 & 0,63 & 0,69 \\
\hline Ecclinusa ramiflora Mart. & 4 & 0,58 & 0,07 & 0,35 & 12 & 0,80 & 0,57 & 0,46 \\
\hline Hyeronima alchorneoides Allemão & 2 & 0,29 & 0,18 & 0,89 & 8 & 0,53 & 0,57 & 0,59 \\
\hline Brosimum rubescens Taub. & 4 & 0,58 & 0,03 & 0,16 & 12 & 0,80 & 0,51 & 0,37 \\
\hline Luehea divaricata Mart. \& Zucc. & 3 & 0,43 & 0,04 & 0,21 & 12 & 0,80 & 0,48 & 0,32 \\
\hline
\end{tabular}




\section{COMPOSIÇÃO FLORÍSTICA E ESTRUTURA DE UM FRAGMENTO FLORESTAL EM ÁREA \\ ECOTONAL CERRADO-PANTANAL}

Tabela 2. Lista das espécies arbóreas ocorrentes na área do córrego da cabeceira Rio das Mortes (RM), em área Cerrado-Pantanal em ordem decrescente de índice de valor de importância (IVI\%), município de Santo Antônio do Leverger - MT, 2011.

\begin{tabular}{|c|c|c|c|c|c|c|c|c|}
\hline Espécie & DA & DR & DoA & DoR & FA & FR & IVI (\%) & IVC (\%) \\
\hline Enterolobium schomburgkii (Benth.) Benth. & 3 & 0,43 & 0,04 & 0,21 & 12 & 0,80 & 0,48 & 0,32 \\
\hline Diospyros guianensis (Aubl.) Gürke & 3 & 0,43 & 0,02 & 0,11 & 12 & 0,80 & 0,44 & 0,27 \\
\hline Xylopia emarginata Mart. & 3 & 0,43 & 0,02 & 0,11 & 12 & 0,80 & 0,44 & 0,27 \\
\hline Piper sp. & 3 & 0,43 & 0,01 & 0,06 & 12 & 0,80 & 0,43 & 0,24 \\
\hline Cecropia hololeuca Miq. & 3 & 0,43 & 0,01 & 0,06 & 12 & 0,80 & 0,43 & 0,24 \\
\hline Vochysia divergens Pohl & 2 & 0,29 & 0,07 & 0,34 & 8 & 0,53 & 0,39 & 0,31 \\
\hline Unonopsis sp. & 3 & 0,43 & 0,03 & 0,15 & 8 & 0,53 & 0,37 & 0,29 \\
\hline Cecropia pachystachya Trécul & 3 & 0,43 & 0,02 & 0,10 & 8 & 0,53 & 0,35 & 0,27 \\
\hline Himatanthus lancifolius (Müll. Arg.) Woodson & 3 & 0,43 & 0,02 & 0,10 & 8 & 0,53 & 0,35 & 0,27 \\
\hline Protium pilosum (Cuatrec.) Daly & 2 & 0,29 & 0,04 & 0,20 & 8 & 0,53 & 0,34 & 0,24 \\
\hline Pourouma sp. & 2 & 0,29 & 0,02 & 0,10 & 8 & 0,53 & 0,31 & 0,19 \\
\hline Virola sebifera Aubl. & 2 & 0,29 & 0,02 & 0,10 & 8 & 0,53 & 0,31 & 0,19 \\
\hline Apuleia leiocarpa (Vogel) J.F.Macbr. & 1 & 0,14 & 0,10 & 0,50 & 4 & 0,27 & 0,30 & 0,32 \\
\hline Erythroxylum sp. & 3 & 0,43 & 0,01 & 0,06 & 4 & 0,27 & 0,25 & 0,24 \\
\hline Apocynaceae 1 & 1 & 0,14 & 0,07 & 0,35 & 4 & 0,27 & 0,25 & 0,24 \\
\hline Micropholis sp. & 1 & 0,14 & 0,05 & 0,26 & 4 & 0,27 & 0,22 & 0,20 \\
\hline Enterolobium timbouva Mart. & 2 & 0,29 & 0,01 & 0,06 & 4 & 0,27 & 0,20 & 0,17 \\
\hline Unonopsis guatterioides (A.DC.) R.E.Fr. & 2 & 0,29 & 0,01 & 0,06 & 4 & 0,27 & 0,20 & 0,17 \\
\hline Lacistema polystachium Schnizl. & 1 & 0,14 & 0,02 & 0,11 & 4 & 0,27 & 0,17 & 0,12 \\
\hline Eriotheca gracilipes (K.Schum.) A.Robyns & 1 & 0,14 & 0,01 & 0,06 & 4 & 0,27 & 0,15 & 0,10 \\
\hline Apocynaceae 2 & 1 & 0,14 & 0,01 & 0,06 & 4 & 0,27 & 0,15 & 0,10 \\
\hline Calophyllum brasiliense Cambess. & 1 & 0,14 & 0,01 & 0,06 & 4 & 0,27 & 0,15 & 0,10 \\
\hline Campomanesia eugenioides (Cambess.) D.Legrand ex Landrum & 1 & 0,14 & 0,01 & 0,06 & 4 & 0,27 & 0,15 & 0,10 \\
\hline Pourouma cecropiifolia Mart. & 1 & 0,14 & 0,01 & 0,06 & 4 & 0,27 & 0,15 & 0,10 \\
\hline Bauhinia sp. & 1 & 0,14 & 0,01 & 0,06 & 4 & 0,27 & 0,15 & 0,10 \\
\hline Matayba guianensis Aubl. & 1 & 0,14 & 0,00 & 0,01 & 4 & 0,27 & 0,14 & 0,07 \\
\hline Total & 694 & 100 & 20 & 100 & 1508 & 100 & 100 & 100 \\
\hline
\end{tabular}

Cabe salientar que, de acordo com IBGE (2012), áreas de tensão ecológica guardam suas próprias características ecológicas, o que faz com que essas áreas geralmente apresentem maior diversidade biológica e também espécies endêmicas.

As famílias de maior representatividade com relação ao número de indivíduos foram: Fabaceae 106 indivíduos $(15,25 \%)$, Lauraceae 68 indivíduos $\quad(9,78 \%), \quad$ Burseraceae 68 indivíduos (9,78\%), Rubiaceae 46 indivíduos $(6,61 \%)$, Melastomataceae 41 indivíduos
$(5,89 \%)$, Sapotaceae 37 indivíduos $(5,32 \%)$, Chrysobalanaceae 32 indivíduos (4,60\%), Lythraceae 31 indivíduos (4,46\%), Siparunaceae 27 indivíduos $(4,88 \%)$, e Annonaceae 26 indivíduos (3,74\%). O valor da abundância dessas famílias representou 69,4\%, ou seja, 482 dos 695 indivíduos na comunidade (Tabela 1).

A família Fabaceae é bem representativa em estudos realizados no Cerrado (FELFILI et al., 2007; entre outros). A expressividade dessa família é marcante em levantamentos que consideram a baixa 
condição de fertilidade natural dos solos, atribuída possivelmente à grande capacidade apresentada por várias espécies de fixação de nitrogênio (SILVA et al., 2004; ARAÚJO et al., 2009).

É importante registrar a presença do gênero Inga, pertencente à família Fabaceae, participando com duas espécies (Inga sp. e Inga vera Willd.), ocorrendo na $9^{\mathrm{a}}$ e $11^{\mathrm{a}}$ posições na área de estudo. De acordo com Mata \& Felix (2007) e Araújo et al. (2009), a maioria das espécies desse gênero são encontradas em formações de diferentes domínios vegetacionais sendo consideradas generalistas e de grande importância na recomposição e recuperação de áreas degradadas e florestas ciliares e estabilização de solos ácidos.

As famílias Araliaceae, Chrysobalanaceae, Euphorbiaceae e Myrsinaceae apresentaram duas espécies cada, as demais famílias apresentaram apenas uma espécie cada (Tabela 1). A porcentagem de espécies que apresentaram apenas um exemplar por hectare foi $18 \%$, correspondendo a 14 espécies do total e os gêneros Xylopia (Annonaceae) e Miconia (Melastomataceae) foram os mais diversificados apresentando três espécies cada (Tabela 1). A concentração de espécies em poucas famílias, neste caso, as dez famílias representando $59,0 \%$ da riqueza, tem sido relatada em outros trabalhos no Bioma Cerrado: Silva et al. (2002), em Goiás; Felfili et al. (2002), em Mato Grosso; e Assunção \& Felfili (2004) no Distrito Federal. Ferreira Júnior et al. (2008), avaliando a composição e diversidade florística em área de tensão ecológica na região de contato floresta ombrófila/floresta estacional, também obtiveram resultados similares. Ressalta-se, que a presença de muitas outras famílias e espécies pouco representadas evidencia a diversidade na área em estudo.

\section{As espécies Tapirira guianensis}

Aubl.e Buchenavia tomentosa Eichler ocupam $2^{\mathrm{a}}$ e $6^{\mathrm{a}}$ posições, respectivamente, em função dos elevados valores de dominância absoluta (Tabela 2). Neste sentido, Araújo et al. (2009) enfatizam que os valores da dominância têm pouca influência na classificação do valor de importância das espécies amostradas, visto que as de maiores valores de importância apresentam elevada densidade e/ou frequência, indicando que se encontram presentes em toda área estudada.

As quinze espécies mais importantes da comunidade, tomando-se como base o valor de importância foram: Ocotea olivacea A.C.Sm., T. guianensis, 


\section{COMPOSIÇÃO FLORÍSTICA E ESTRUTURA DE UM FRAGMENTO FLORESTAL EM ÁREA ECOTONAL CERRADO-PANTANAL}

Protium heptaphyllum (Aubl.) Marchand, Physocalymma scaberrimum Pohl, Sloanea guianensis (Aubl.) Benth., B. tomentosa, Protium pilosissimum Engl., Cordia alliodora (Ruiz \& Pav.) Cham., Inga sp., Licania humilis Cham. \& Schltdl., Ormosia arborea (Vell.) Harms, Inga vera, Siparuna guianensis Aubl., Chrysophyllum gonocarpum (Mart. \& Eichler ex Miq.) Engl. e Miconia sp.. Essas espécies foram responsáveis por $52,0 \%$ do valor de importância (VI) total (Tabela 2).

Ressalta-se que a espécie $O$. olivaceae apresenta maior VI na área em estudo e é relatada por Quinet et al. (2016) como de ocorrência registrada apenas nos Estados do Acre, Amazonas e Rondônia, já foi registrada por COPEL (2012) no Estado de Mato Grosso.

Pode-se afirmar que a área estudada caracteriza-se por apresentar a maior parte

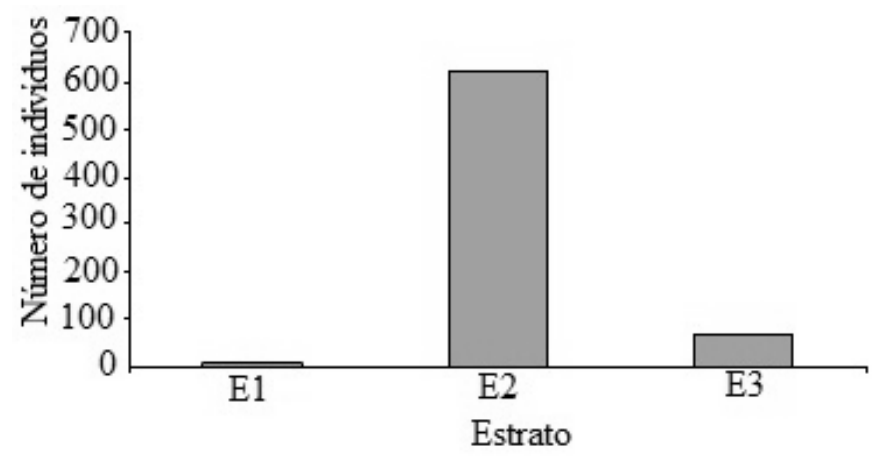

Figura 1. Número de indivíduos por estrato de altura (n/ha), em que: E1 = estrato 1 (ht < 2,9 m); $\mathrm{E} 2=$ estrato $2(2,9 \mathrm{~m} \leq \mathrm{ht}<14,6 \mathrm{~m}) ; \mathrm{E} 3=$ estrato $3(\mathrm{ht} \geq 14,6 \mathrm{~m})$, na área do córrego da cabeceira do Rio das Mortes, município de Santo Antônio do Leverger, MT, 2011. 
A altura média dos indivíduos foi de 9,0 m, valor superior ao proposto por Ribeiro \& Walter (1998) para a subdivisão cerrado típico (3 a $6 \mathrm{~m}$ ). Entretanto, os valores foram similares aos obtidos por Negrelle (2016) em uma área em Barão de Melgaço, Pantanal de Mato Grosso cuja altura média dos indivíduos arbóreos foi de $8,62 \pm 3,26 \mathrm{~m}$.

Ressalta-se que a concentração de espécies no estrato médio, foi similar aos resultados encontrados tanto por Ribeiro \& Walter (1998) quanto por Duarte (2007), respectivamente, em áreas de cerrado e pantanal, ressaltando-se que os intervalos de classes foram diferentes do que foi adotado neste estudo.

A existência de indivíduos em todos os estratos (inferior, médio e superior) é um indício de sua representatividade na estrutura da comunidade florestal em todas as fases de seu desenvolvimento (HOSOKAWA et al., 1998; SCOLFORO \& MELO, 1997). As que não seguem esta regra, poderão não estar presentes futuramente na comunidade vegetal (HOSOKAWA et al., 1998), por não se reproduzirem ou por não se regenerarem no local, com exceção daquelas que são características dos estratos inferior e médio da floresta (SCOLFORO \& MELO, 1997).

\section{CONCLUSÕES}

A diversidade florística observada foi alta quando comparada à média para os biomas Cerrado e Pantanal, sendo a família Fabaceae aquela que apresentou a maior riqueza de espécies e a família Lauraceae, com uma contribuição expressiva da espécie Ocotea olivacea.

A existência de indivíduos arbóreos em todos os estratos da comunidade constituiu evidência da representatividade na estrutura da comunidade florestal, em todas as fases de seu desenvolvimento.

\section{REFERÊNCIAS}

ANDRADE, L. A. Z.; FELFILI, J. M.; VIOLATTI, L. 2002. Fitossociologia de uma área de cerrado denso na RECOR IBGE, Brasília - DF. Acta Botanica Brasilica, Belo Horizonte, v. 16, n.2, p. 225-240.

APG - ANGIOSPERM PHYLOGENY GROUP. 2009. An update of the Angiosperm Phylogeny Group classification for the orders and families of flowering plants: APG III. Botanical Journal of the Linnean Society, London, v. 161, p. 105-121.

ARAÚJO, R. A. COSTA, R. B.; FELFILI, J. M.; GONÇALVEZ, I. K.; SOUSA, R. A. T. M.; DORVAL, A. 2009. Florística e estrutura de fragmento florestal em área de transição na Amazônia Matogrossense no município de Sinop. Acta Amazonica, Manaus, v. 39, n. 4, p. 865-877.

ASSUNÇÃO, S. L.; FELFILI, J. M. 2004. Fitossociologia de um fragmento de cerrado sensu stricto na APA do Paranoá, 
DF, Brasil. Acta Botanica Brasilica, Belo Horizonte, v.18, n.4, p.903-909.

BRASIL. 2016. Flora do Brasil $2020 \mathrm{em}$ construção. Jardim Botânico do Rio de Janeiro. Disponível em: <http://floradobrasil.jbrj.gov.br/>. Acesso em: 13 Jul. 2016

COPEL - COMPANHIA PARANAENSE DE ENERGIA. UHE Colíder. 2012. Complementação do inventário florestal, levantamentos florísticos em áreas de cerrados e florestas estacionais e censo de árvores isoladas. Curitiba: COPEL. 481p. Disponível em: <http://www.copel.com/uhecolider/sitear quivos2.nsf/arquivos/inventario_florestal _complementar/\$FILE/Invent\%C3\%A1ri o\%20Florestal\%20Complementar.pdf> Acesso em: 12. Jul. 2016

BRASIL. Departamento Nacional de Produção Mineral. Projeto RADAMBRASIL. 1982. Levantamento de Recursos Naturais. Folha Cuiabá (SD-21). Rio de Janeiro: DNPM.

FELFILI, J. M.; NOGUEIRA, P. E.; SILVA JÚNIOR, M. C.; MARIMON, B. S.; DELITTI, W. B. C. 2002. Composição florística e fitossociologia do cerrado sentido restrito no município de Água Boa-MT. Acta Botanica Brasilica, Belo Horizonte, v. 16, n. 1, p. 103-112.

FELFILI, J. M.; NASCIMENTO, A. R. T.; FAGG, C. W.; MEIRELLES, E. M. 2007. Floristic composition and community structure of a seasonally deciduous forest on limestone outcrops in Central Brazil. Revista Brasileira de Botânica, São Paulo, v.30, n.4, p.611-621.

FERREIRA JÚNIOR, E. V.; SOARES, T. S.; COSTA, M. F. F.; SILVA V. S. M. 2008. Composição, diversidade e similaridade florística de uma floresta tropical semidecídua submontana em Marcelândia - MT, Brasil. Acta Botanica Brasilica, Belo Horizonte, v.38, n.4, p.673-680.
GUARIM, V. L. M. S.; MORAES, E. C. C.; PRANCE, G. T.; RATTER, J. A. 2000. Inventory of a Mesotrophic Callisthene Cerradão in the Pantanal of Mato Grosso, Brazil. Edinburg Journal of Botany, Edinburgh, v.3, p.429-436.

HAIDAR, R. F.; FAGG, J. M. F.; PINTO, J. R. R.; DIAS, R. R.; DAMASCO, G.; SILVA, L. C. R.; FAGG, C. W. 2013. Florestas estacionais e áreas de ecótono no estado do Tocantins, Brasil: parâmetros estruturais, classificação das fitofisionomias florestais e subsídios para conservação. Acta Amazônica, Manaus, v. 43, n. 3, p.261-290.

HOSOKAWA, R. T.; MOURA, J.B.; CUNHA, U.S. 1998. Introdução ao manejo e economia de florestas. Curitiba: UFPR. 162p.

IBGE - INSTITUTO BRASILEIRO DE GEOGRAFIA E ESTATÍSTICA. 2012. Manual técnico da vegetação brasileira. 2 ed. Rio de Janeiro: IBGE. 275p.

IBGE - INSTITUTO BRASILEIRO DE GEOGRAFIA E ESTATÍSTICA. 2004. Mapa dos Biomas do Brasil. Disponível em: <http://www.ibge.gov.br/home/geocienci as>. Acesso em: 16. Fev. 2010.

LIMA JÚNIOR, G. A.; OLIVEIRA, C. R. M.; RAGGI, F.; ARMOND, C.; MEIRA NETO, J. A. A.; VALENTES, G. 2007. Fitofisionomia, espectro biológico e valores de cobertura em um campo cerrado da FLONA de Paraopeba, Minas Gerais. Revista Brasileira de Biociências, Porto Alegre, v. 5, supl. 2, p. 684-686, p. 1-3.

MARIMON JUNIOR, B. H.; HARIDASAN, M. 2005. Comparação da vegetação arbórea e características edáficas de um cerradão e um cerrado sensu stricto em áreas adjacentes sobre solo distrófico no leste de Mato Grosso, Brasil. Acta Botanica Brasilica, Belo Horizonte, v.19, n. 4, p. 913-926. 
MATA, M. F.; FELIX, L. P. 2007. Flora da Paraíba, Brasil: Inga Mill. (Leguminosaemimosoideae). Revista Brasileira de Biociências, Porto Alegre, v. 5, supl. 2, p. 135-137.

MORAIS, R. F.; SILVA, E. C. S.; METELO, M. R. L.; MORAIS, F. F. 2013. Composição florística e estrutura da comunidade vegetal em diferentes fitofisionomias do Pantanal de Poconé, Mato Grosso. Rodriguésia, Rio de Janeiro, v. 64, n. 4, p. 775-790.

MORELLATO, P. C.; LEITÃO FILHO, H. F. (Orgs.). 1995. Ecologia e preservação de uma floresta tropical urbana: Reserva de Santa Genebra. Campinas: UNICAMP. 136p.

MULLER-DOMBOIS, D.; ELLENBERG, H. 1974. Aims and methods in vegetation ecology. New York: John Wiley and Sons. 574p.

NEGRELLE, R. R. B. 2016. Composição e estrutura do componente arbóreo de mata com acuri no Pantanal Matogrossense, Brasil. Ciência Florestal, Santa Maria, v. 26, n. 2, p. 589-600.

NEIFF, J. J. 2003. Planícies de inundação são ecótonos? In: HENRY, R. (Org.). Ecótonos nas interfaces dos ecossistemas aquáticos. São Carlos: Rima. p. 29-46.

ODUM, E. 2010. Ecologia. Rio de Janeiro: Guanabara-Koogan. 434p.

QUINET, A.; BAITELLO, J. B.; MORAES, P. L. R.; ASSIS, L.; ALVES, F. M. Lauraceae. In: Lista de Espécies da Flora do Brasil. Jardim Botânico do Rio de Janeiro. Disponível em: <http://floradobrasil.jbrj.gov.br/jabot/flor adobrasil/FB23408>. Acesso em: $21 \mathrm{Jul}$. 2016

RIBEIRO J. F.; WALTER, B. M. T. 1998. Fitofisionomias do bioma cerrado. In: SANO, S.M.; ALMEIDA, S.P. (Ed.). Cerrado: ambiente e flora. Planaltina: EMBRAPA-CPAP, p. 89-166.
RODRIGUES, N. B.; LIMA JÚNIOR, G. A.; COSTA, R. B.; SILVA, F. H. B.; CUNHA, C. N. 2012. Composição florística, fitossociologia e diversidade de uma Floresta Estacional Decidual em Cáceres, Pantanal de Mato Grosso. Multitemas, Campo Grande, n. 41, p. 1933.

SCOLFORO, J. R. S.; MELO, J. M. 1997. Inventário florestal. Lavras: UFLA/FAEPE. 341p.

SILVA, L. O.; COSTA, D. A.; SANTO FILHO, K. E.; FERREIRA, H. D.; BRANDÃO, D. 2002. Levantamento florístico e fitossociológico em duas áreas de cerrado sensu stricto no Parque Estadual da Serra de Caldas Novas, Goiás. Acta Botanica Brasilica, Belo Horizonte, v. 16, n. 1, p. 43-53, p.1-12.

SILVA, N. R. S.; MARTINS, S. V.; MEIRA NETO, J. A. A. 2004. Composição florística e estrutura de uma Floresta Estacional Semidecidual Montana em Viçosa, MG. Revista Árvore, Viçosa, v. 28, n. 3, p. 397-405.

SILVA, R. A.; PAIXÃO, E. C.; CUNHA, C. N.; FINGER, Z. 2016. Fitossociologia da comunidade arbórea de cerrado sensu stricto do Parque Nacional da Chapada dos Guimarães Nativa, Sinop, v. 4, n. 2, p. 82-86.

SILVEIRA, E. P.; COSTA, R. B.; FELFILI, J. M. 2009. Florística da vegetação remanescente de Cerrado sensu stricto em terra indígena no noroeste de Mato Grosso, Brasil. Revista de Biologia Neotropical, Goiânia, v. 6, n. 2, p.15-25. VELOSO, H. P.; RANGEL FILHO, A. L. R.; LIMA, J. C. A. 1991. Classificação da vegetação brasileira, adaptada a um sistema universal. Rio de Janeiro: IBGE, $112 \mathrm{p}$.

Recebido em: 18/8/2015 Aceito para publicação em: 9/8/2016 